\title{
Coherence in Microchip Traps
}

\author{
Philipp Treutlein $*$ Peter Hommelhoff \\ Max-Planck-Institut für Quantenoptik und Sektion Physik der \\ Ludwig-Maximilians-Universität, Schellingstr.4, 80799 München, Germany
}

(Dated: October 29, 2018)

\begin{abstract}
We report the coherent manipulation of internal states of neutral atoms in a magnetic microchip trap. Coherence lifetimes exceeding $1 \mathrm{~s}$ are observed with atoms at distances of $5-130 \mu \mathrm{m}$ from the microchip surface. The coherence lifetime in the chip trap is independent of atom-surface distance within our measurement accuracy, and agrees well with the results of similar measurements in macroscopic magnetic traps. Due to the absence of surface-induced decoherence, a miniaturized atomic clock with a relative stability in the $10^{-13}$ range can be realized. For applications in quantum information processing, we propose to use microwave near-fields in the proximity of chip wires to create potentials that depend on the internal state of the atoms.
\end{abstract}

PACS numbers: 32.80.Pj, 39.90.+d, 03.67.Lx, 06.30.Ft

Keywords: magnetic microtraps, atom chip, coherence, atomic clocks, quantum gates

Magnetic microchip traps provide one of the few available techniques for manipulating neutral atoms on the micrometer scale, and the only technique so far that enables nonperiodic, built-to-purpose micron-sized potentials 11. The on-chip creation of Bose-Einstein condensates [2, 3] and the highly controlled manipulation of atomic motion in 'atomic conveyor belts', waveguides, and thermal beam splitters [1] are examples of the versatility of such 'atom chips'. Due to these possibilities, chip traps are promising candidates for the implementation of quantum gates [4], quantum simulations [5], and interferometric sensors [6]. The ability to manipulate superpositions of internal states of the trapped atoms is essential for most of these applications. In quantum information processing (QIP), two internal states $|0\rangle$ and $|1\rangle$ of the atom serve as qubit states. To perform gate operations, long coherence lifetimes of the superposition states $\alpha|0\rangle+\beta|1\rangle$ are required and therefore decoherence processes have to be avoided. Atoms in chip traps, however, can potentially suffer from a reduction of the coherence lifetime due to interaction with the surface of the chip [7], in addition to other decoherence mechanisms which are also present in macroscopic traps [8].

In this letter, we demonstrate coherent manipulation of internal atomic states in a magnetic microchip trap. We create superpositions of two hyperfine ground states of ${ }^{87} \mathrm{Rb}$ atoms in a thermal ensemble close to quantum degeneracy and perform Ramsey spectroscopy to determine the coherence lifetime (Fig. (1). With atoms at distances of $5-130 \mu \mathrm{m}$ from the surface of the chip, we observe coherence lifetimes exceeding $1 \mathrm{~s}$. These lifetimes are independent of the atom-surface distance, and agree well with those observed in macroscopic magnetic traps [8].

The observed robustness of the superposition states is an extremely encouraging result for atom chip applications in QIP and opens a new perspective on applications in precision metrology. We demonstrate an atomic clock in the chip trap and measure the relative stability of its transition frequency. Our measurements show that a portable atom chip clock with a relative stability in the $10^{-13} \tau^{-1 / 2} / \sqrt{\mathrm{Hz}}$ range is a realistic goal.

To realize the collisional phase gate proposed in [4], a state-selective potential is needed. We point out that state selectivity for our state pair can be provided by microwave potentials. These potentials, considered in the early 90s [9, 10] but abandoned later, gain new actuality as near-field traps on atom chips.

To achieve long coherence lifetimes with magnetically trapped atoms in the proximity of the chip surface, we choose the $\left|F=1, m_{F}=-1\right\rangle \equiv|0\rangle$ and $\mid F=2, m_{F}=$ $1\rangle \equiv|1\rangle$ hyperfine levels of the $5 S_{1 / 2}$ ground state of ${ }^{87} \mathrm{Rb}$. The magnetic moments of the two states are approximately equal. At a magnetic field of $B_{0} \sim 3.23 \mathrm{G}$, both states experience the same first-order Zeeman shift and the remaining magnetic field dependence of the transition frequency $\nu_{10}$ is minimized [8]. In all of our experiments, we therefore adjust the field in the center of the trap to $B_{0}$. This greatly reduces spatial inhomogeneities of $\nu_{10}$ in the trap, since both states experience the same potential to good approximation. Furthermore, superpositions of this state pair are particularly robust against decoherence due to magnetic field noise. Thermal magnetic near-field noise has been predicted to be a relevant

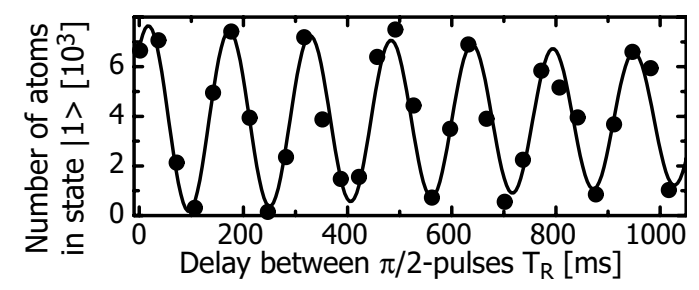

FIG. 1: Ramsey spectroscopy of the $|0\rangle \leftrightarrow|1\rangle$ transition with atoms held at a distance $d=9 \mu \mathrm{m}$ from the chip surface. An exponentially damped sine fit to the Ramsey fringes yields a $1 / e$ coherence lifetime of $\tau_{c}=2.8 \pm 1.6 \mathrm{~s}$. Each data point corresponds to a single shot of the experiment. 

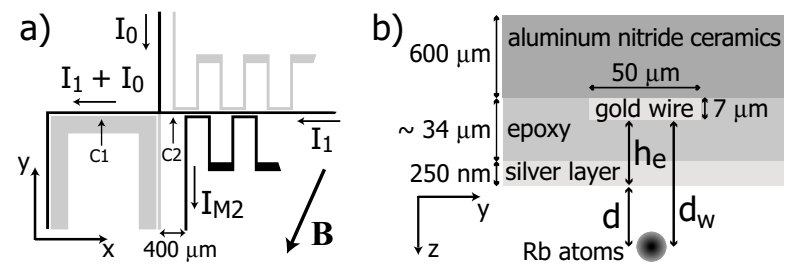

FIG. 2: (a) Layout of the relevant wires on the chip substrate. $\mathrm{C} 1$ : position of the initial magnetic trap. C2: position of the 'measurement trap' used in the experiments. (b) Layer structure of the substrate. Current is carried by gold wires $h_{e} \sim 27 \mu \mathrm{m}$ below the silver surface. $d$ denotes the atomsurface distance, while $d_{w}$ refers to the atom-wire distance.

atom-surface coupling mechanism in the regime of distances studied here [7]. For our state pair, this source of decoherence is suppressed by more than $10^{6}$ (the squared ratio of electron and nuclear magnetic moment) and we expect surface decoherence rates to be negligible.

Our experimental setup has been previously described [3, 11]. The magnetic potentials are created by sending currents through microscopic gold conductor patterns on a substrate and superposing a homogeneous bias field (Fig. 21). The preparation of the atomic ensemble proceeds in a multi-step sequence involving loading of the microtrap from a mirror-MOT, compression of the trap and evaporative cooling [3]. By the end of this sequence, a Ioffe-type 'measurement trap' centered at the position C2 in Fig. 22 contains a thermal atomic ensemble of typically $1.5 \times 10^{4}$ atoms in state $|0\rangle$ at a temperature of $0.6 \mu \mathrm{K}$. The measurement trap is created by the currents $I_{0}, I_{1}$, and $I_{M 2}$ and the bias field $\mathbf{B}=\left(B_{x}, B_{y}, 0\right)$ shown in Fig. 2a. By adjusting all three currents and $B_{y}$, the atoms can be placed at distances $d=0-130 \mu \mathrm{m}$ from the chip surface (see Fig. 2b) with only small changes in the shape of the magnetic potential. For each experimentally studied distance, the field $\mathbf{B}_{\min }$ at the center of the trap was calibrated spectroscopically [8] and set to $\left|\mathbf{B}_{\min }\right|=B_{0}$ by adjusting $B_{x}$. Typical experimental parameters are $I_{0}=500 \mathrm{~mA}, I_{1}=120 \mathrm{~mA}, I_{M 2}=700 \mathrm{~mA}$, $B_{y}=-5.50 \mathrm{G}$, and $B_{x}=-2.18 \mathrm{G}$, leading to trap frequencies $\left(f_{x}, f_{y}, f_{z}\right)=(50,350,410) \mathrm{Hz}$ at $d=9 \mu \mathrm{m}$. The atoms are held in the measurement trap while the coherent internal state manipulation is performed. After the manipulation, the trap is switched off within $150 \mu$ s and the atoms are detected after a time of flight of typically $4 \mathrm{~ms}$. Atoms are detected by absorption imaging on the $F=2 \rightarrow F^{\prime}=3$ transition. This allows direct determination of the number of atoms in state $|1\rangle, N_{1}$. To alternatively determine the number of atoms in state $|0\rangle$, $N_{0}$, we first blow away all atoms in $|1\rangle$ with the resonant probe light. The $|0\rangle$ atoms are then optically pumped to $\left|F=2, m_{F}=+2\right\rangle$ and imaged as before.

Coherent internal state manipulation is achieved by coupling $|0\rangle$ and $|1\rangle$ through a two-photon microwave- radio frequency transition. The microwave frequency $\nu_{\mathrm{mw}}$ is detuned $1.2 \mathrm{MHz}$ above the $\left|F=2, m_{F}=0\right\rangle$ intermediate state and radiated from a sawed-off waveguide outside the vacuum chamber. The radio frequency $\nu_{\mathrm{rf}}$ is either applied to the same external coil that is used for evaporative cooling, or to a wire on the chip. $\nu_{\mathrm{mw}}$ and $\nu_{\text {rf }}$ are phase locked to an ultrastable $10 \mathrm{MHz}$ quartz os-

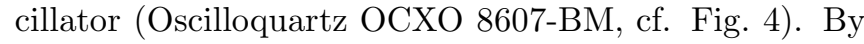
applying the two-photon drive for a variable time and detecting the number of atoms transferred to $|1\rangle$, we observe Rabi oscillations with a resonant two-photon Rabi frequency of $\sim 0.5 \mathrm{kHz}$. In this way, single-qubit rotations can be realized. The $\pi$-pulse transition probability is $N_{1} /\left(N_{0}+N_{1}\right)=95 \pm 5 \%$.

To test for decoherence of the superposition states, we perform Ramsey spectroscopy: The atoms in state $|0\rangle$ are held in the measurement trap for a time $T_{H}$ before a first $\pi / 2$-pulse creates a superposition of $|0\rangle$ and $|1\rangle$. After a delay $T_{R}$, a second $\pi / 2$-pulse is applied, and the resulting state is probed. Time-domain Ramsey fringes are recorded by varying $T_{R}$ while keeping $\Delta_{R}=\nu_{\mathrm{mw}}+\nu_{\mathrm{rf}}-\nu_{10}$ fixed $\left(\Delta_{R} \ll \nu_{10} \simeq 6.8 \mathrm{GHz}\right)$. Alternatively, frequency-domain Ramsey fringes are recorded by scanning $\Delta_{R}$ with constant $T_{R}$. Loss of coherence can show up in different ways in the Ramsey signal. A spatial variation of $\nu_{10}$ across the atomic ensemble leads to a decay of the fringe contrast, while temporal fluctuations of $\nu_{10}$ lead to increasing phase noise as $T_{R}$ is increased.

Figure 1 shows time-domain Ramsey fringes. The number of atoms detected in state $|1\rangle$ oscillates at the frequency difference $\Delta_{R}=6.4 \mathrm{~Hz}$, while the interference contrast decays with a coherence lifetime of $\tau_{c}=$ $2.8 \pm 1.6 \mathrm{~s}$. The measurement of Fig. [1 was performed at $d=9 \mu \mathrm{m}$ from the room-temperature chip surface. In [8], similar coherence lifetimes are reported for the same state pair, but with atoms in a macroscopic magnetic trap, far away from any material objects. This suggests that atom-surface interactions indeed do not limit the coherence lifetime in our experiment.

To further probe for surface effects, we study decoherence as a function of atom-surface distance $d$ (Fig. 3). At each distance, we record frequency-domain Ramsey oscillations for several values of $T_{R}$ and determine the contrast $C\left(T_{R}\right)$ of each oscillation. Figure 3 shows the result for $T_{R}=50 \mathrm{~ms}$ and $T_{R}=1 \mathrm{~s}$. Within the experimental error, the contrast does not show a dependence on atom-surface distance. Additionally, we have compared the signal-to-noise ratio $S / N$ of the interference signals at different $d$. We typically observe $S / N=6$ for $T_{R}=1 \mathrm{~s}$, where $S$ is the peak-to-peak amplitude of the sinusoidal fit to the Ramsey oscillation and $N$ is the standard deviation of the fit residuals over one oscillation period. $S / N$ is independent of $d$ within experimental error, indicating that the processes causing amplitude and phase fluctuations of the interference signal do not depend on atom-surface distance on this time scale. 


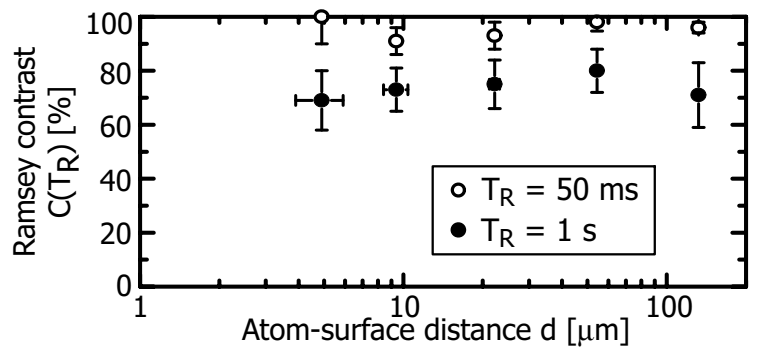

FIG. 3: Contrast $C\left(T_{R}\right)$ of the Ramsey fringes as a function of atom-surface distance $d$ for two values of the time delay $T_{R}$ between the $\pi / 2$-pulses. For each data point, $C\left(T_{R}\right)=\left(N_{\max }-\right.$ $\left.N_{\min }\right) /\left(N_{\max }+N_{\min }\right)$ was obtained from a sinusoidal fit to frequency-domain Ramsey fringes. $N_{\max }\left(N_{\min }\right)$ is the maximum (minimum) of the oscillation in $N_{1}$. The data points for $d=(5,9,22,54,132) \mu \mathrm{m}$ were measured with atomic ensembles of temperatures $T=(0.2,0.6,0.7,0.6,0.3) \mu \mathrm{K}$ and peak densities $n_{0}=(4,3,1,1,5) \times 10^{12} \mathrm{~cm}^{-3}$.

The observed decoherence is mainly due to a combination of the residual differential Zeeman shift and the density-dependent collisional shift of $\nu_{10}$ across the ensemble [8]. Consequently, we observe a dependence of $\tau_{c}$ on the temperature $T$ and on the peak density $n_{0}$ of the atoms. To avoid systematic errors, we have checked that there is no systematic variation of $T$ and $n_{0}$ as $d$ is varied (see Fig. 3). The observed noise on the Ramsey oscillation is mostly phase noise and can be attributed to ambient magnetic field fluctuations (see below). For $T_{R}>1 \mathrm{~s}$ the phase noise increases and obscures the oscillation even before the contrast has completely vanished.

To calibrate the atom-surface distance $d=d_{w}-h_{e}$ (see Fig. 20), we use two methods: For $d>10 \mu \mathrm{m}$, we apply a technique described in [12] in which the imaging beam is slightly tilted towards the chip surface. For $d<10 \mu \mathrm{m}$, the distance $d$ can no longer be resolved by our imaging system. In this case, $d_{w}$ can be determined from a simulation of the trapping potential, but $d$ is affected by uncertainties in $h_{e}$, which was not well-controlled during manufacture. To determine $h_{e}$, we move the atoms close to the surface and measure the remaining atom fraction after $10 \mathrm{~ms}$ as a function of $d_{w}$ 15. Using a model for atom loss due to the attractive Casimir-Polder surface potential [15], which contains $h_{e}$ as the only free parameter, we determine $h_{e}=27.1 \mu \mathrm{m}$ with a statistical error of $0.1 \mu \mathrm{m}$. Including errors in the model parameters, such as trap frequencies and temperature, we estimate an uncertainty in $d$ of $\pm 1 \mu \mathrm{m}$ (error bars in Fig. 33).

Thermal magnetic field noise driving spin-flip transitions to untrapped states [7] and surface evaporation have been observed to limit the lifetime $\tau_{N}$ of the atomic population near a surface [13, 14, 15]. We also observe these effects. The trap lifetime in state $|0\rangle$ decreases from $\tau_{N}=11 \mathrm{~s}$ for $d>20 \mu \mathrm{m}$ to $\tau_{N}=1.6 \mathrm{~s}$ for $d=5 \mu \mathrm{m}\left(\tau_{N}\right.$ for state $|1\rangle$ is slightly lower due to stronger coupling to the surface [7] and dipolar relaxation). For $d<5 \mu \mathrm{m}$,

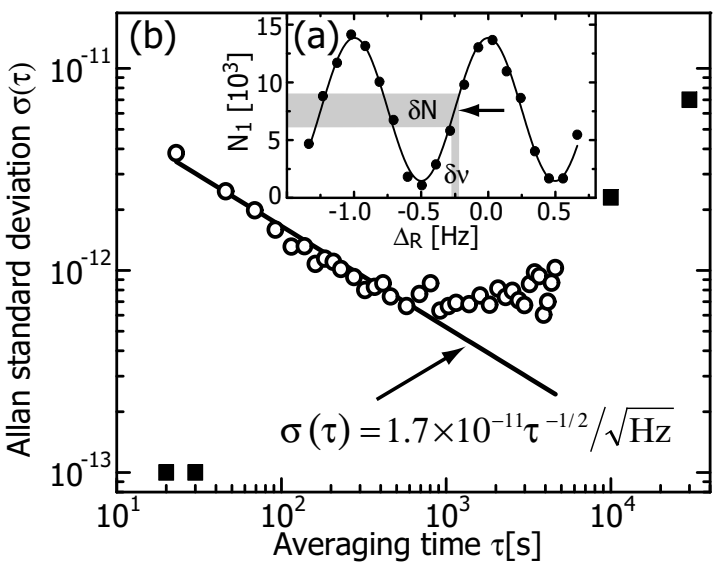

FIG. 4: (a) Ramsey resonance for $T_{R}=1 \mathrm{~s}$ in the microtrap at $d=54 \mu \mathrm{m}$ from the surface (solid circles). The line is a sinusoidal fit to the data. The arrow indicates the operating point on the slope of the resonance used in the measurement of $\sigma(\tau)$. Frequency fluctuations $\delta \nu$ lead to fluctuations $\delta N$ in the detected number of atoms. (b) Measured Allan standard deviation $\sigma(\tau)$ of the atomic clock in the microtrap compared to the quartz reference oscillator (open circles). The solid line is a fit with $\sigma(\tau) \propto \tau^{-1 / 2}$ representing the performance of the atomic clock. For $\tau>6 \times 10^{2} \mathrm{~s}$ the drift of the quartz reference becomes apparent. The manufacturers' specification of the quartz stability is shown in solid squares.

this atom loss prohibits coherence measurements with $T_{R} \sim 1 \mathrm{~s}$. To distinguish loss of population from loss of coherence, $T_{H}+T_{R}$ is kept constant during the Ramsey scan by appropriately adjusting the hold time $T_{H}$ for each value of $T_{R}$. Thus, the overall time the atoms spend close to the surface is independent of $T_{R} . N_{0}+N_{1}$ at the time of detection therefore remains approximately constant. The small lifetime difference of the two states reduces $C\left(T_{R}=1 \mathrm{~s}\right)$ by less than $5 \%$.

One motivation for atom chip research is the perspective of creating miniaturized cold-atom devices. Due to the long coherence lifetime, it is natural to consider utilizing the $|0\rangle \leftrightarrow|1\rangle$ transition in an atomic clock on the chip. We demonstrate the principle of such a clock and measure its frequency stability relative to the quartz reference oscillator. Figure 4 i shows frequencydomain Ramsey fringes for $T_{R}=1 \mathrm{~s}$. We set the twophoton drive to the slope of the Ramsey resonance (arrow in the figure), and repeat the experiment many times with a cycle period of $23 \mathrm{~s}$. Any temporal drift $\delta \nu$ of $\nu_{10}$ with respect to the reference will change $\Delta_{R}$ and therefore show up as a variation $\delta N$ of $N_{1}$. From repeated measurements of $\delta N$ we determine the relative frequency fluctuations $\delta \nu / \nu_{10}$. In Fig. 列 we plot the Allan standard deviation $\sigma(\tau)$ [17] of $\delta \nu / \nu_{10}$ as a function of averaging time $\tau$. For short $\tau, \sigma(\tau)$ decreases as $\sigma(\tau)=1.7 \times 10^{-11} \tau^{-1 / 2} / \sqrt{\mathrm{Hz}}$, corresponding to shot-toshot fluctuations of $\delta \nu=24 \mathrm{mHz}$ r.m.s. For $\tau>6 \times 10^{2} \mathrm{~s}$, the long-term drift of the reference leads to a departure 
from the $\tau^{-1 / 2}$ line. We have modeled the frequency fluctuations and can account for the observed value of $\delta \nu$. It is dominated by ambient magnetic field fluctuations of $\sim 5 \mathrm{mG}$. Smaller contributions are due to $\sim 4 \%$ variations in total atom number leading to variations of the collisional shift 8], and due to imperfections of the detection system. We estimate that realistic improvements - magnetic shielding, operation in a shallower trap at lower atomic density, shot-noise limited detection, and a $6 \mathrm{~s}$ cycle (which is realistic in a chip trap 3 ) — will lead to a frequency stability in the $10^{-13} \tau^{-1 / 2} / \sqrt{\mathrm{Hz}}$ range. While this does not reach the stability level of fountain clocks, a chip-based clock has the advantage of a simple, compact and portable setup.

Applications of magnetic microtraps in QIP require long coherence lifetimes of the qubit in the presence of unavoidable magnetic field noise. A state pair with equal magnetic moments is therefore much better suited than any other combination of ground state sublevels. In [4], a phase gate with atoms in a magnetic microtrap was proposed, and a gate time of $0.4 \mathrm{~ms}$ was estimated. Implementing this gate with atoms in states $\{|0\rangle,|1\rangle\}$ located $\sim 5 \mu \mathrm{m}$ above a chip with micron-sized wires, $\sim 10^{3}$ gate operations could be performed before decoherence from magnetic noise occurs. The gate requires state-dependent potentials. However, a combination of static magnetic and electric fields, as considered in [4, 16], does not provide state-selectivity for our state pair, whose magnetic moments and electrostatic polarizabilities are equal. Instead, we propose to apply tailored microwave near-fields and make use of the AC Zeeman effect (the magnetic analog of the AC Stark effect). In ${ }^{87} \mathrm{Rb}, \mathrm{AC}$ Zeeman potentials derive from magnetic dipole transitions near $\omega_{0} / 2 \pi=6.835 \mathrm{GHz}$ between the $F=1$ and $F=2$ hyperfine manifolds of the ground state. The magnetic component of a microwave field of frequency $\omega_{0}+\Delta$ couples the $\left|F=1, m_{F}\right\rangle$ to the $\left|F=2, m_{F}{ }^{\prime}\right\rangle$ sublevels and leads to energy shifts that depend on $m_{F}$ and $m_{F}{ }^{\prime}$. In a spatially varying microwave field, this results in a state-dependent potential landscape.

A microwave trap based on AC Zeeman potentials was proposed in [9] and experimentally demonstrated in [10]. This trap employed microwave radiation in the far field of the source. Due to the centimeter wavelength $\lambda_{\mathrm{mw}}$ of the radiation, field gradients were weak and structuring the potential on the micrometer scale is impossible. In a chip trap, on the other hand, atoms are trapped at distances $d \ll \lambda_{\mathrm{mw}}$ from the chip wires. Thus, they can be manipulated with microwave near fields, generated by microwave currents in the wires, which may be fed from a stripline [18]. In the near field of the currents, the magnetic component of the microwave field has the same position dependence as a static magnetic field created by equivalent dc currents. In this way, state-dependent $\mathrm{AC}$ Zeeman potentials varying on the micrometer scale can be created. In combination with state-independent static magnetic traps, the potential geometries required for QIP can be realized.

To be specific, we consider a static-field trap at $d=$ $10 \mu \mathrm{m}$ from an additional chip wire carrying a microwave current of $20 \mathrm{~mA}_{\mathrm{pp}}$. The wire is oriented such that the magnetic component of the microwave field at the position of the atoms is polarized parallel to the local static magnetic field. The microwave couples $|0\rangle \leftrightarrow \mid F=$ $\left.2, m_{F}=-1\right\rangle$ and $\left|F=1, m_{F}=1\right\rangle \leftrightarrow|1\rangle$ with identical resonant Rabi frequencies $\Omega_{R} / 2 \pi=2.4 \mathrm{MHz}$. The Zeeman splitting due to the static field (a few $\mathrm{MHz}$ ) prevents two-photon transitions to other sublevels driven by polarization impurities. For $\Delta / 2 \pi=50 \mathrm{MHz}, \Omega_{R} \ll \Delta$ and the coupling changes the static magnetic moment of the qubit states by only $\sim 10^{-4} \mu_{\mathrm{B}}$ such that both states still experience the same static-field potentials. The microwave, on the other hand, leads to a differential energy shift of $|0\rangle$ and $|1\rangle, U_{\mathrm{mw}} \simeq \hbar \Omega_{R}^{2} / 2 \Delta=h \cdot 58 \mathrm{kHz}$, sufficiently large for the state-selective manipulation required in QIP. Besides this application, AC Zeeman potentials can be used to create microtraps for atoms in hyperfine sublevels such as $m_{F}=0$, which cannot be trapped in a static magnetic trap.

In conclusion, we have performed coherent internal state manipulation in a magnetic microchip trap with coherence lifetimes exceeding $1 \mathrm{~s}$ at distances down to $5 \mu \mathrm{m}$ from the chip surface. This paves the way for a variety of applications, most notably chip-based quantum gates and atomic clocks.

We thank T. Udem and M. Zimmermann for the crystal reference oscillator. Work supported in part by the EU's IST program (ACQP, IST-2001-38863).

* E-mail philipp.treutlein@physik.uni-muenchen.de

$\dagger$ Present address: Varian Physics 220, Stanford University, Stanford, CA 94305, U.S.A.

[1] J. Reichel, Appl. Phys. B 75, 469 (2002), R. Folman et al., Adv. At. Mol. Opt. Phys. 48, 263 (2002).

[2] H. Ott et al., Phys. Rev. Lett. 87, 230401 (2001).

[3] W. Hänsel et al., Nature 413, 498 (2001).

[4] T. Calarco et al., Phys. Rev. A 61, 022304 (2000).

[5] E. Jané et al., Quant. Inf. Comp. 3, 15 (2003).

[6] M.A. Kasevich, Science 298, 1363 (2002).

[7] C. Henkel et al., Appl. Phys. B 76, 173 (2003).

[8] D.M. Harber et al., Phys. Rev. A 66, 053616 (2002).

[9] C.C. Agosta et al., Phys. Rev. Lett. 62, 2361 (1989).

[10] R.J.C. Spreeuw et al., Phys. Rev. Lett. 72, 3162 (1994).

[11] J. Reichel et al., Phys. Rev. Lett. 83, 3398 (1999).

[12] S. Schneider et al., Phys. Rev. A 67, 023612 (2003).

[13] M.P.A. Jones et al., Phys. Rev. Lett. 91, 080401 (2003).

[14] D.M. Harber et al., J. Low Temp. Phys. 133, 229 (2003).

[15] Y. Lin et al., Phys. Rev. Lett. 92, 050404 (2004).

[16] P. Krüger et al., Phys. Rev. Lett. 91, 233201 (2003).

[17] G. Santarelli et al., Phys. Rev. Lett. 82, 4619 (1999).

[18] T.C. Edwards, Foundations for microstrip circuit design (John Wiley \& Sons, Chichester, 1981). 